\title{
Umprogrammierte T-Zellen nutzen
}

Die Therapie mit modifizierten, gegen CD19 gerichteten T-Zellen erwies sich in der Behandlung eines rezidivierten oder refraktären akuter lymphoblastischer Leukämie (ALL) als wirksam und führte zu einer hohen Remissionsrate, selbst bei Patienten, bei denen die Stammzelltransplantation nicht angeschlagen hatte.

Für die T-Zell-Therapie werden T-Zellen so modifiziert, dass sie chimäre Antigenrezeptoren, die gegen CD19 gerichtet sind, exprimieren. Diese zytotoxischen TLymphozyten (CTL019) können dann Zellen angreifen, die das Antigen CD19 tragen. Nun wurde das Verfahren an 30 Kindern und Erwachsenen mit rezidivierter oder refraktärer ALL untersucht.

$90 \%$ der 30 Patienten hatten bei der ersten Untersuchung, die einen Monat nach der CTL019-Infusion stattfand, eine vollständige Remission, darunter zwei Patienten, deren Erkrankung gegenüber Blinatumomab refraktär war, und $15 \mathrm{~Pa}$ tienten, die sich einer Stammzelltransplantation unterzogen hatten.

Nur bei drei Patienten war eine minimale Resterkrankung nachweisbar. Bei sieben Patienten mit vollständiger Remission trat innerhalb von 8,5 Monaten nach der Infusion ein Rezidiv auf. Insgesamt blieben 19 Patienten in Remission, 15 von ihnen erhielten keine weitere Therapie.

Nach sechs Monaten lagen das ereignisfreie Überleben bei $67 \%$ und das Gesamtüberleben bei $78 \%$. Sieben Patienten starben aufgrund einer Krankheitsprogression oder eines Rezidivs. Die Wahrscheinlichkeit, dass die CTL019-Zellen

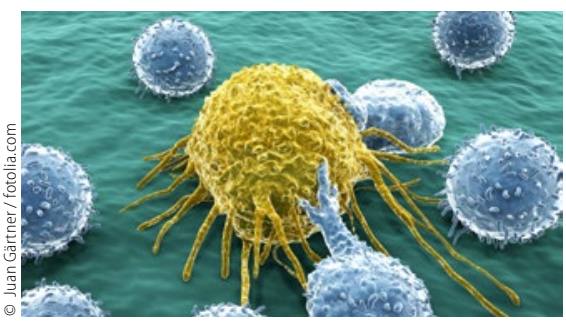

Neue Strategien lenken T-Lymphozyten gezielt zu den Krebszellen.

persistierten, betrug nach sechs Monaten $68 \%$, die Wahrscheinlichkeit für eine rezidivfreie B-Zell-Aplasie $73 \%$.

Bei allen Patienten entwickelte sich ein Zytokin-Release-Syndrom, das mit dem Anti-Interleukin-6-Antikörper Tocilizumab behandelt werden konnte.

Judith Neumaier

Maude SL et al. Chimeric Antigen Receptor T Cells for Sustained Remissions in Leukemia. N Engl J Med. 2014;371(16):1507-17.

\section{Erhaltungstherapie bei multiplem Myelom}

\section{Keine Lebensverlängerung nachweisbar}

Bei Patienten mit einem multiplen Myelom lässt sich durch eine Erhaltungstherapie mit Thalidomid oder Lenalidomid das progressionsfreie Überleben verlängern. Ein Einfluss auf das Gesamtüberleben ist dagegen nicht erkennbar, wie die Ergebnisse einer Metaanalyse vermuten lassen. US-Onkologen haben in dieser die Daten von 18 Studien mit 7.730 Patienten untersucht.

In sechs randomisierten und kontrollierten Phase-III-Studien erfolgte die Erhaltungstherapie mit Lenalidomid, in den übrigen Studien mit Thalidomid. Lenalidomid wurde teilweise mit Prednison oder Dexamethason kombiniert, Thalidomid u. a. auch mit Bortezomib oder Interferon alpha.

Die Auswertungen zeigten, dass die Immunmodulation mit Thalidomid oder Lenalidomid in der Erhaltungstherapie in allen Studien signifikant wirksamer war als ein Regime ohne Erhaltungstherapie oder ohne einen der beiden Immunmodulatoren. Die Wahrscheinlichkeit, ohne Fortschreiten der Erkrankung weiterzuleben, wurde durch die Erhaltungstherapie auf Basis von Thalidomid oder Lenalidomid um $38 \%$ erhöht (Hazard Ratio [HR] 0,62; $<<0,001)$.

Keinen signifikanten Unterschied konnten die Onkologen dagegen im Zusammenhang mit dem Parameter Gesamtüberleben zwischen den Verumund Kontrollgruppen feststellen (HR 0,93; $\mathrm{p}=0,082$ ).

Die Behandlung mit den Immunmodulatoren ist jedoch wie erwartet mit einer erhöhten Rate an vaskulären Grad-3/4Nebenwirkungen assoziiert, nämlich mit pulmonalen Embolien und tiefen Venenthrombosen. Ähnlich gesteigert wurde die Rate an peripheren Neuropathien, Neutropenien und Thrombozytopenien. Vor allem die höhere Wahrscheinlichkeit für Neuropathien ist bei der Entscheidung für eine derartige Erhaltungstherapie zu berücksichtigen.

Peter Leiner

Wang Y et al. Maintenance Therapy With Immunomodulatory Drugs in Multiple Myeloma: A Meta-Analysis and Systematic Review. J Natl Cancer Inst. 2015; Nov 18 [Epub ahead of print].

\section{kurz notiert}

\section{Astrozytome bilden Kommunikationsnetzwerke}

Astrozytome sprechen schlecht auf Standardtherapien an. Eine Ursache für diese Resistenz könnte ihre Fähigkeit sein, Kommunikationsnetzwerke auszubilden, wie ein internationales Forscherteam herausfand [Osswald M et al. Nature. 2015;528(7580):93-8]. Die Tumorzellen bilden Microtubes aus, die sie mit anderen Tumorzellen verbinden. Die Schnittstellen des Netzwerks werden durch Connexin 43 erzeugt, an der Ausbildung der Microtubes ist das Protein GAP-43 beteiligt. Über dieses Netzwerk können die Tumorzellen dann untereinander kommunizieren, ins Gehirn einwandern, proliferieren und Reparaturmechanismen in Gang zu setzen. Möglicherweise ist dies auch ein Weg wie sie zum Beispiel Schäden an Tumorzellen beseitigen, die durch eine Strahlentherapie entstehen.

Um die Erfolge bei der Behandlung von Astrozytomen zu verbessern, wäre es sinnvoll, die Kommunikation der Astrozytome untereinander zu blockieren. Mögliche Behandlungsstrategien könnten daher die medikamentöse Hemmung des Tumorzellnetzwerks durch Kalziumblocker oder Substanzen, die Connexin-43 oder GAP-43 beeinflussen, sein. 